\title{
Predicación secundaria en español: representación y análisis sintáctico-semántico ${ }^{1}$
}

\author{
Secondary predication in Spanish: syntactic semantic representation and analysis
}

\author{
Marcos Cárdenas ${ }^{2}$
}

\section{Resumen}

La predicación corresponde a un fenómeno semántico que se proyecta en la sintaxis de la lengua y permite establecer la relación primaria sujeto-predicado para la expresión del significado a nivel oracional. Se distingue además un tipo de predicación secundaria que se establece entre elementos internos que selecciona o no el predicado principal. La representación y análisis sintáctico de la predicación secundaria se ha abordado desde la gramática generativa tradicional en la cual, sin embargo, existen miradas divergentes que la conciben como una cláusula mínima y como una categoría funcional. El objetivo de este artículo es contrastar estas perspectivas y analizar su pertinencia desde los puntos de vista descriptivo y explicativo en el marco de un enfoque sintáctico-semántico. En vista de su relevancia en la expresión del significado, se concluye que la predicación como categoría funcional permite dar cuenta de una función única que integra operaciones primarias y secundarias jerárquicamente. Al final del artículo, se discute sobre el potencial predictivo de la predicación secundaria en español en torno a su representación a nivel neurolingüístico.

Palabras clave: complemento predicativo, adjunto, Sintagma Predicado, cláusula mínima

\begin{abstract}
Predication corresponds to a semantic phenomenon which projects onto syntax and allows to establish the primary subject-predicate relationship for the expression of meaning at the sentence level. It is also known that another type of predication can be established between the internal elements that are selected by the main predicate. Syntactic representation and analysis of secondary predication has been mainly studied from the traditional generative grammar approach; nevertheless, there still exist diverging perspectives that consider secondary predication as a reduced clause and as a functional category. The purpose of this paper is to compare these perspectives and analyze their descriptive and explanatory adequacy from a syntacticsemantic approach. In the light of its relevance in the expression of meaning we conclude that predication as a functional category allows to account for a single function that integrates primary and secondary operations in a hierarchical way. At the end of this paper we also discuss the predictive potential of secondary predication in Spanish at the neurolinguistic level.
\end{abstract}

Keywords: predicative complement, adjunct, Predicate Phrase, reduced clause

\footnotetext{
${ }^{1}$ Trabajo presentado en curso del programa de Doctorado en Lingüística, PUCV. El autor cuenta con el apoyo de la beca Conicyt para doctorado nacional.

${ }^{2}$ Docente de la Escuela de Educación de la Universidad de O'Higgins (UOH), Rancagua, Chile. Correo: marcos.cardenas@uoh.cl
} 


\section{Introducción}

La predicación es una función gramatical que en términos formales se ha asociado unívocamente a la relación sujeto-predicado en oraciones. Sin embargo, su rol en la organización de la gramática se extiende al grado de interdependencia que se establece entre los elementos presentes en la estructura oracional de acuerdo con requisitos de selección semántica específicos. A partir de un enfoque generativista (Chomsky, 1965, 1981), es posible sostener que esta función es la encargada de asignar papeles temáticos a los argumentos que selecciona un predicado verbal para completar su expresión. Lo descrito anteriormente se considera un principio en la lingüística generativa tradicional, no obstante, no es suficiente para describir y explicar la relación interna de otros constituyentes como en el caso de (1) donde descalza se clasifica formalmente como un elemento opcional y de (2) donde loco se describe como un elemento obligatorio:

(1) María retrató a la modelo descalza.

(2) Los niños volvieron loco al profesor.

La descripción generativista formal de ambos elementos en español ha sido abordada principalmente por Demonte y Masullo (1999), quienes adscriben a la noción de cláusulas mínimas, a saber, cláusulas que carecen de flexión gramatical, para describir este elemento. Dada la naturaleza funcional de la predicación, Bowers $(1993,2001)$ postula como alternativa la existencia de una categoría funcional Pr cuyo propósito es explicar las relaciones predicativas que se establecen en la oración. Aunque ambas propuestas se enmarcan en la gramática generativa tradicional e intentan dar cuenta de la misma función gramatical, divergen conceptualmente en cuanto a cómo esta se representa y analiza sintácticamente, en particular, en casos de predicación secundaria.

A partir de este planteo, nos proponemos contrastar ambas propuestas en términos de su adecuación descriptiva y explicativa en torno a la predicación secundaria en español. Junto con ello, discutiremos sobre qué tipo de predicciones sintáctico-semánticas permite el estudio experimental de esta estructura desde una perspectiva neurolingüística.

$\mathrm{El}$ artículo se organiza de la siguiente manera. En las secciones 1 y 2, se presenta el marco teórico que sustenta el análisis del trabajo. En la sección 3, se presenta la discusión en vista del análisis previo e implicancias teórico-prácticas. Finalmente, en 4, se presentan las conclusiones derivadas del trabajo.

\section{Marco teórico}

\subsection{La noción de predicación}

La predicación ha sido abordada fundamentalmente desde perspectivas léxico-sintácticas (Chomsky, 1981), léxico-semánticas (Rothheim, 2011) y sintáctico-semánticas (Bowers, 1993, 2001; Demonte \& Masullo, 1999; cf. Gross, 2013) para dar cuenta del lugar que ocupa en la organización de la gramática. En la tradición filosófica, Aristóteles describió este fenómeno como una proposición que posee una estructura binaria compuesta por un sujeto que hace referencia a una entidad y un predicado que expresa una propiedad de dicha entidad (Espinal, 2014). Con la irrupción de la gramática generativa (Chomsky, 1965, 1981) y su potencial descriptivo y explicativo, fue posible establecer cómo se relacionan estos componentes en términos del lugar que ocupan en la estructura argumental y son representados en la forma lógica (FL), lo cual ha permitido distinguir formalmente entre argumentos o elementos seleccionados 
por el predicado y adjuntos o elementos no seleccionados por el predicado. En el modelo generativista clásico, la predicación se concibe como una función que asigna papeles temáticos a los argumentos que exige el predicado (Chomsky, 1981). Al respecto, Espinal sostiene que "un predicado es una función que necesita saturarse mediante uno o más argumentos, los cuales tendrán que ocupar las posiciones sintácticas adecuadas para recibir los papeles temáticos correspondientes" (2014, p. 188). Por ejemplo, en (1) El niño rompió el vidrio el predicado romper selecciona dos argumentos (el niño y el vidrio) para que se satisfagan los requisitos de selección semántica que se encuentran codificados en su valencia biargumental. Una forma de codificar las restricciones de selección es mediante el uso de relaciones temáticas que describen el rol del argumento con respecto al predicado. Por un lado, el sintagma determinante (SD) el niño constituye el argumento externo que recibe el papel temático de agente y cuyo predicado es todo el sintagma verbal (SV) rompió el vidrio; por otro lado, el sintagma determinante (SD) el vidrio constituye el argumento interno directo que recibe el papel temático de tema y cuyo predicado es el núcleo léxico romper.

\section{O [SD El niño [SV rompió [SD el vidrio]]]}

Según esta perspectiva, dichas relaciones deben ajustarse a ciertos criterios para que su representación en la forma lógica (FL) sea legítima y cumpla con las condiciones de buena formación. En este caso, el criterio temático o criterio- $\theta$ (Chomsky, 1981; Carnie, 2002) establece que i. a cada argumento se le asigna solo un papel temático y que ii. cada papel temático se asigna solo a un argumento. De este modo, podemos señalar que el SV rompió el vidrio es el predicado del SD el niño, relación por la cual se establece una predicación primaria como se visualiza en (2):

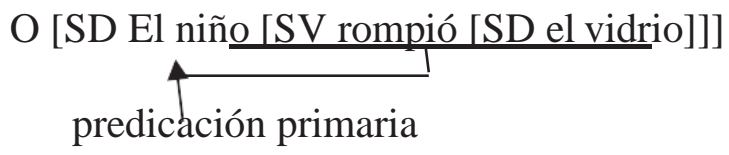

En este caso, la relación entre SD y SV se expresa mediante el núcleo del Sintagma Flexión -ió que funciona como anclaje temporal para que la estructura argumental se configure de modo tal que satisfagan el criterio temático y el de selección léxica. En esta visión, la flexión gramatical se considera como el eje vertebrador entre el SD sujeto y el SV predicado y su proyección máxima es la oración o sintagma flexión (SFlex) (Espinal, 2014; Carnie, 2002), transformándose así en el núcleo endocéntrico de la categoría funcional.

\subsection{Predicación no verbal en español}

Además de la predicación primaria verbal descrita anteriormente, existen predicados no verbales con núcleos léxicos preposicionales $(\mathrm{P})$, adjetivales $(\mathrm{A})$ y nominales $(\mathrm{N})$ que han suscitado mucho interés investigativo dada la información aspectual que aportan a la estructura (Schultze-Berndt, 2017), por ejemplo, oraciones causativas del tipo (3) María hizo muy feliz a Juan, donde la estructura interna del verbo causativo hacer implica un cambio en el estado de cosas y el adjetivo feliz se orienta hacia el argumento interno directo a Juan. Así, es de suponer que existe otro tipo de relación -además de la predicación primaria entre el SV hizo muy feliz a Juan y el SD Maríaque se establece entre el SP a Juan y el SA muy feliz y cuya representación sintáctica según el modelo generativista tradicional está condicionada por el criterio temático y el 


\section{Marcos Cárdenas}

principio de proyección extendida (PPE) (Chomsky, 1981; Carnie, 2002). Para dar respuesta a lo anterior, Demonte y Masullo (1999) proponen que el SA muy feliz es un complemento predicativo seleccionado semánticamente por el verbo causativo hacer y que está orientado hacia el argumento interno directo, a saber, el SP a Juan. Al respecto, agregan que el SA corresponde a un complemento obligatorio, pues, si se elimina, cambia el sentido de la oración y a su vez está inserto en una cláusula mínima (CM), es decir, una oración que carece de flexión temporal (Espinal, 2014). Por su parte, en la propuesta de Bowers (1993, 2001), el SA muy feliz corresponde también a un elemento seleccionado por el predicado principal (hacer) y su relación se concibe como una predicación secundaria obligatoria. En contraste a Demonte y Masullo que consideran este tipo de predicación como una CM, Bowers señala que el SA muy feliz corresponde más bien al complemento de un núcleo predicativo (Pr) que se proyecta en un sintagma predicado (SPr).

En el siguiente apartado, nos referimos a la noción y análisis de la predicación secundaria desde la perspectiva generativista de Demonte y Masullo (1999) y Bowers (1993, 2001). En particular, nos referimos a cómo se representan sintácticamente las relaciones de predicación primaria y secundaria en oraciones que presentan ambos casos.

\subsection{El complemento predicativo como cláusula mínima (CM)}

Demonte y Masullo sostienen que una oración simple es la realización sintáctica de una proposición semántica "que expresa un estado o acontecimiento en el que están implicados uno o varios participantes [...] seleccionados o exigidos por las acciones o estados" (1999, p. 2463). Estos participantes son seleccionados por un predicado verbal en la medida que se satisfacen ciertas condiciones de formación. Aquellos elementos que no cumplen con la condición de selección léxica que exige el predicado principal pasarán a ser adjuntos u opcionales. Los complementos predicativos pueden clasificarse según esta propuesta teórica en complementos predicativos seleccionados léxicamente y complementos predicativos no seleccionados léxicamente o adjuntos. Al respecto, Demonte y Masullo agregan que "los complementos predicativos son aquellos constituyentes que modifican simultáneamente al predicado verbal y a un sintagma nominal de la misma oración (típicamente, al sujeto y al objeto directo sintáctico" (1999, p. 2463). En cuanto a su naturaleza sintáctica, los complementos predicativos pueden no verse afectados por la pronominalización del sintagma que modifican como se muestra en (5):

Juan guardó la camisa sucia.

Juan la guardó sucia.

Además, pueden separarse del nombre que modifican y luego aparecer junto al verbo del que se predica como en (6):

Juan guardó sucia la camisa.

Pueden a su vez anteponerse a la oración como focalizadores contrastivos o tematizadores como se muestra en (7):

(7) Sucia guardó Juan la camisa. (focalizador)

Sucia es como Juan guardó la camisa (tematizador)

Asimismo, los predicativos pueden mantener su posición en el SV de oraciones pasivas como: 


\section{Marcos Cárdenas}

(8) La camisa fue guardada sucia.

Los rasgos descritos anteriormente dan cuenta de un constituyente o sintagma que realiza una función sintáctica independiente y que cumple con las condiciones de buena formación que exige el modelo. Del mismo modo, es posible establecer claramente dos clases de complementos predicativos: los seleccionados y los no seleccionados por el predicado principal.

La primera clase se refiere a elementos que se adjuntan a un SN y este último es un constituyente obligatorio que forma parte de la valencia del verbo principal. La tabla 1 muestra la clasificación de los adjuntos según su comportamiento sintáctico. Los predicados descriptivos o depictivos realizados por un adjetivo denotan un estado transitorio de la entidad cuando se realiza la acción. Por ejemplo, desganada se refiere al estado de la soprano cuando cantó. En los pseudoresultativos, los requisitos argumentales del verbo pintar exigen un argumento agente cuya acción resulte en un cambio transitorio del estado del argumento interno.

Tabla 1. Clases de complementos predicativos no seleccionados léxicamente o adjuntos.

\begin{tabular}{|l|l|l|}
\hline \multicolumn{3}{|l|}{ Complementos predicativos adjuntos o no seleccionados } \\
\hline $\begin{array}{l}\text { Predicativos } \\
\text { descriptivos }\end{array}$ & Orientado al sujeto & e.g. La soprano cantó desganada. \\
\cline { 2 - 3 } $\begin{array}{l}\text { Predicativos } \\
\text { pseudoresultativos }\end{array}$ & e.g. Mi hermana pintó la casa roja. \\
\hline
\end{tabular}

La segunda clase corresponde a constituyentes que son exigidos por el verbo principal, por lo tanto, no se pueden elidir, pero no anular y se comportan como complementos obligatorios. De este modo, "completan el sentido del verbo, determinando junto con él el papel semántico de los argumentos nominales relacionados con ellos" (Demonte \& Masullo, 1999, p. 2498). La tabla 2 describe los criterios de clasificación de los complementos predicativos seleccionados léxicamente y ejemplos de cada caso:

Tabla 2. Clases de complementos predicativos seleccionados léxicamente.

\begin{tabular}{|c|c|c|}
\hline \multicolumn{3}{|c|}{ Complementos predicativos seleccionados } \\
\hline \multirow{2}{*}{$\begin{array}{l}\text { En construcciones } \\
\text { transitivas complejas } \\
\text { o con CM }\end{array}$} & $\begin{array}{l}\text { En CM de verbos } \\
\text { epistémicos, } \\
\text { volitivos y de } \\
\text { orientación } \\
\text { prospectiva }\end{array}$ & $\begin{array}{l}\text { e.g. Encuentro tu propuesta muy original. } \\
\text { Juan considera a María inteligente. }\end{array}$ \\
\hline & $\begin{array}{l}\begin{array}{r}\text { En CM exigidas por } \\
\text { verbos }\end{array} \\
\text { causativos }\end{array}$ & e.g. Los alumnos volvieron loco al profesor. \\
\hline $\begin{array}{l}\text { En oraciones con } \\
\text { verbos soporte }\end{array}$ & \multicolumn{2}{|c|}{ e.g. Tiene una mirada amable. } \\
\hline \multirow{2}{*}{$\begin{array}{l}\text { Seleccionados por } \\
\text { verbos intransitivos }\end{array}$} & $\begin{array}{l}\text { De verbos } \\
\text { Pseudocopulativos }\end{array}$ & e.g. Su hijo cayó enfermo. \\
\hline & $\begin{array}{l}\text { Introducidos por } \\
\text { preposición }\end{array}$ & e.g. Juan presume de feliz. \\
\hline
\end{tabular}


La predicación secundaria admite la presencia de una flexión de concordancia nominal como se ejemplifica en (9) y (10), donde los subíndices i y j representan relaciones predicativas:

(9) Maríai llegó cansadai. (cf. María llegó.)

(cansada, adjunto predicativo, no seleccionado por el predicado principal).

(María se predica de llegó cansada, corresponde a predicación primaria).

(10) Juan considera $a_{i}$ a María inteligente j. (cf. Juan considera a María.)

(inteligente, complemento predicativo, seleccionado por el predicado principal)

(Juan se predica de considera a María inteligente, corresponde a predicación primaria).

En (9) cansada no representa un atributo inherente de María, sino un estado temporal que se relaciona más bien con la forma en que María llegó, es decir, María estaba cansada cuando llegó. En (10) inteligente satisface los rasgos léxicos que exige el verbo para completar su significado y así imponer en conjunto criterios de selección que el argumento externo (sujeto sintáctico) debe satisfacer para recibir el papel de experimentante [+animado].

Como se señaló en la primera parte, el elemento que vincula el SD sujeto con el SV predicado en la tradición generativista es la flexión temporal cuya proyección máxima es SFlex. En vista de ello, se propone que la predicación secundaria optativa puede representarse como una oración mínima o cláusula mínima (Demonte \& Masullo, 1999; Espinal, 2014) que carece de flexión temporal y que se adjunta a la proyección máxima u oración. En la figura 1 se esquematizan las relaciones predicativas tanto primaria (SD FLEX SV) como secundaria (CM adjunta a SFlex). En la primera, el SV asigna el papel de agente al SDi. En la segunda, el constituyente PROi recibe el papel de experimentante por parte del SA desganada. La CM se representa como un adjunto de la oración (SFlex).

Figura 1. Representación sintáctica de predicación secundaria optativa (adjunto predicativo)



Por su parte, en la figura 2 se esquematiza una relación de predicación secundaria donde $\mathrm{V}$ manda-c a CM y sus hijas SD y SA. La CM es un constituyente obligatorio que recibe el papel temático de tema proposicional por parte del predicado principal considerar (cf. Juan considera que María es inteligente), función que se ejecuta en paralelo al proceso de saturación semántica del complemento predicativo con respecto al SD objeto directo interno. En conjunto SV y CM asignan el papel temático de experimentante al SD sujeto.

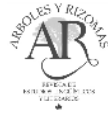


Figura 2. Representación sintáctica de predicación secundaria obligatoria (complemento predicativo)

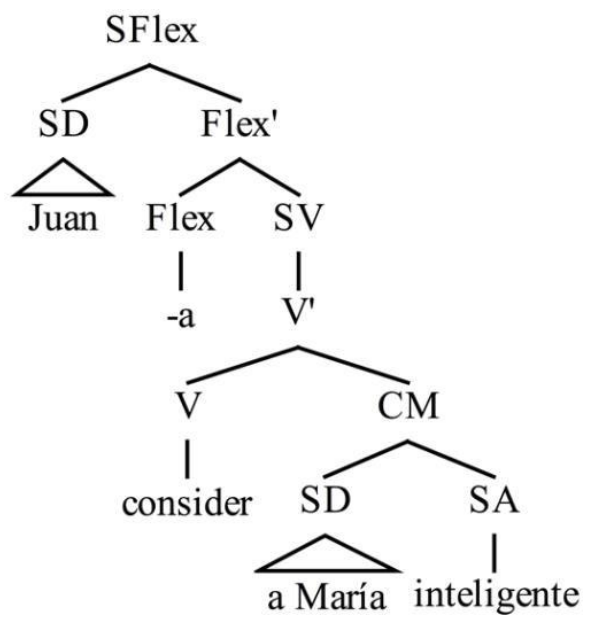

En la visión de Demonte y Masullo (1999), la predicación secundaria se representa sintácticamente como una cláusula mínima que carece de flexión temporal. Su inserción como complemento predicativo en la oración depende de los criterios de selección que exige el predicado principal, pero también de los rasgos que presenta el elemento que ocupa la posición de especificador en la estructura. De no cumplirse los criterios semánticos de selección, la cláusula mínima se representa como un adjunto predicativo de la proyección máxima. Así, se desprende que la predicación secundaria obligatoria y opcional es una función gramatical específica que se ejecuta bajo condiciones sintácticas y semánticas acotadas en comparación a la predicación primaria que rige la organización total de la estructura oracional.

\subsection{Sintagma predicado $(\mathrm{SPr})$}

Bowers $(1993,2001)$ concibe la predicación como la función más importante en la gramática. Su propuesta parte del supuesto de la lingüística generativa tradicional de que un predicado es una expresión no saturada que se debe combinar con una expresión de entidad para formar una proposición; en otras palabras, un predicado selecciona argumentos a los cuales asigna papeles temáticos para completar la expresión del estado o proceso que denota. Esta definición semántica de predicación se sustenta en que una función gramatical como la predicación no se puede explicar por medio de reglas transformacionales o simple combinatoria de elementos (Chomsky, 1965), especialmente, en casos de cláusulas mínimas, pues existe una estructura que subyace a la secuencia de constituyentes de la superficie, como en el siguiente ejemplo:
Ella considera a Juan un buen amigo.
Ella dio a Juan un lindo regalo.
(a) $[\mathrm{SN}+\mathrm{V}+\mathrm{SP}+\mathrm{SN}]$
(b) $[\mathrm{SN}+\mathrm{V}+\mathrm{SP}+\mathrm{SN}]$

Aparentemente, ambas secuencias son idénticas. Sin embargo, (11a) posee un predicado verbal que exige un elemento obligatorio (un buen amigo) que a su vez se predica del argumento interno (a Juan) para saturar su expresión. Si se elimina este elemento, cambia el sentido de la oración. Por su parte, (11b) presenta una secuencia idéntica, pero la selección y orden de los constituyentes depende exclusivamente de la valencia triargumental del predicado dar.

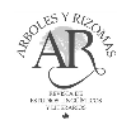


En la misma línea, Bowers realiza una observación similar respecto del tratamiento de elementos que presentan casos de ambigüedad. Como se observa en (12), una misma secuencia puede tener distintas interpretaciones:

(12) La mujer pintó a la modelo descalza.

(a) la mujer está descalza.

(b) la modelo está descalza.

La interpretación de (12) depende si el SA descalza se predica del sujeto mujer o del objeto modelo, y su descripción como adjunto no explica cómo se relaciona con el resto de los constituyentes. Es a partir de estas observaciones que Bowers sugiere que debe de existir tanto una relación estructural entre constituyentes que defina la relación sujeto-predicado y que la distinga de otras relaciones de adyacencia, así como una forma de representar las relaciones predicativas entre constituyentes distantes como en (12). Dicha observación también se extiende a los requisitos de formación que impone la categoría SFlex, por ejemplo, que el sujeto de una cláusula principal se sitúe en la posición de especificador de una categoría funcional F, lo cual exige aparentemente que se asuma la misma estructura en las cláusulas mínimas (Bowers, 2001).

Dado lo anterior, Bowers propone que una teoría de la predicación debiese considerar ambos tipos de predicación como una función única cuya operación esté regida por principios mínimos generativistas como los de rección y de papeles temáticos (Chomsky, 1981) y que cumplan con las condiciones estructurales que imponen versiones recientes de la Teoría de X con Barra (Carnie, 2002). En su argumentación, Bowers reconoce la universalidad del SFlex y de la categoría funcional Flex, y adhiere además a la hipótesis del sujeto interno al SV "que nos permite concebir el argumento externo del verbo como un sujeto antes de concordar" (Bosque \& Gutiérrez, 2009 , p. 259) para dar cuenta de relaciones predicativas primarias. De este modo, y para explicar la naturaleza interna de las cláusulas reducidas, Bowers (1993) propone dividir la categoría Flex en F y Pr, en la que F representa la función deíctica de Flex mientras que Pr representa su función predicativa como se visualiza en la figura 3 :

Figura 3. Representación sintáctica de predicación primaria y secundaria (SFlex y SPr)
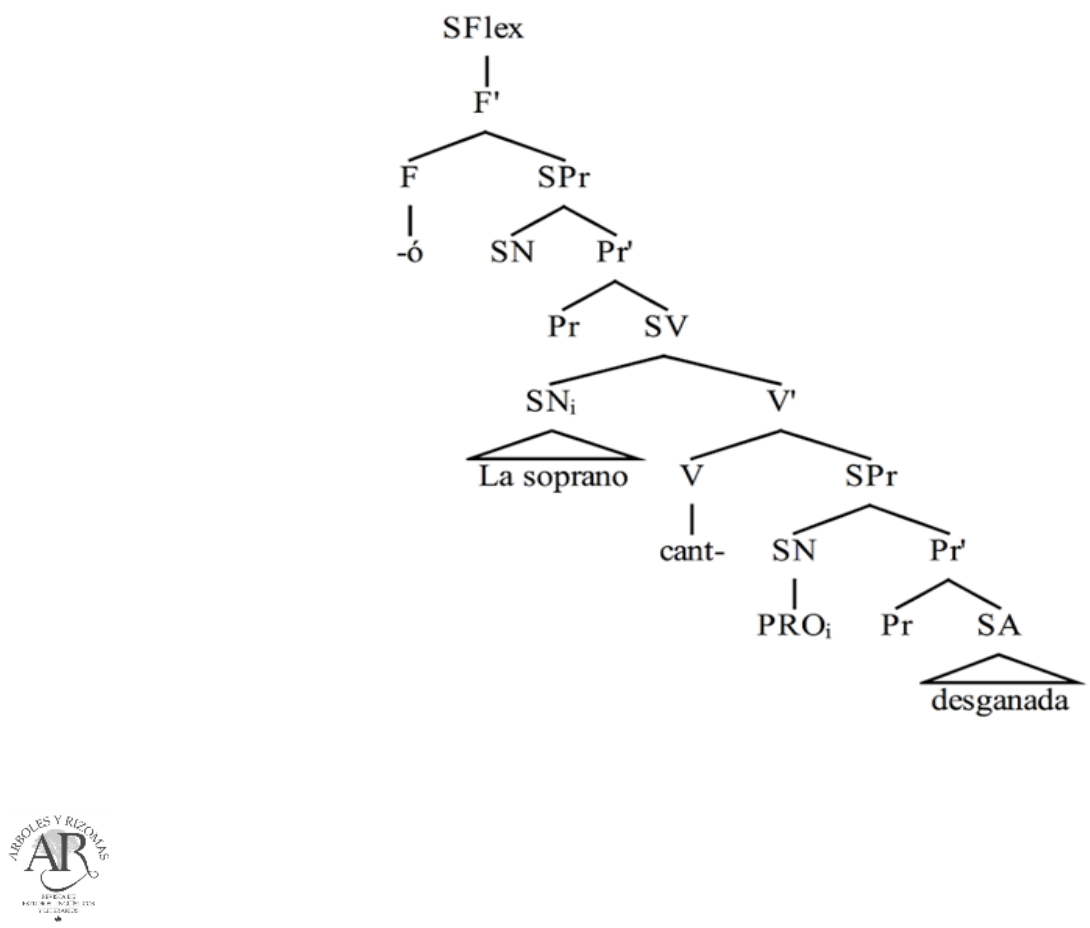
Bowers (1993) arguye que "syntactically, Pr is a functional category that selects the maximal projection XP of any lexical category $\mathrm{X}$ and whose maximal projection PrP can either be generated independently (yielding SC predication) or selected by T (yielding MC predication)" (p. 302). Según esta definición, la figura 3 muestra relaciones predicativas tanto primaria como secundaria, donde: a. F es la función deíctica temporal y especificador del SPr, el que a su vez corresponde a la proyección máxima de $\operatorname{Pr}$ y establece la relación predicativa principal; b. el sujeto interno la soprano se sitúa en posición de especificador (Esp) con respecto a V' en el SV;

c. SV selecciona como complemento predicativo el SPr cuya estructura interna presenta un sujeto PROi (SNi) en posición de especificador y proyecta el núcleo funcional Pr que corresponde a la huella del verbo elidido en la cláusula mínima. Este último caso corresponde a predicación secundaria optativa o adjunción. Al respecto, Bowers (2001) agrega que la representación de dependencias sintácticas como el adjunto de la figura 3 se explica en términos del supuesto que PROi está controlado por el antecedente más cercano que lo manda-c (la soprano), y el depictivo descalza adjunto a Pr' se orienta al sujeto externo (la soprano).

Además del caso anterior, existe evidencia adicional que sustenta la propuesta de Bowers en cuanto a la representación de construcciones sintácticas que presentan predicación secundaria obligatoria y verbos epistémicos como considerar. En la figura 4, se visualiza la representación sintáctica del complemento predicativo inteligente. A diferencia del ejemplo de la figura 3, el SA es seleccionado por el predicado principal. La relación entre el SA inteligente y su referente se remite a una condición de rección, al igual que en la figura 3. En este caso, el antecedente inmediato que manda-c a PROj es María. Paralelamente, PROj manda-c a SA inteligente. Es importante destacar que el predicado verbal del SPr secundario ha sido elidido, sin embargo, se interpreta como la cópula ser. Asimismo, el predicado principal se desplaza a su posición superficial de núcleo predicativo, dejando una huella en la estructura profunda. De este modo, ambos tipos de predicación se integran jerárquicamente en la estructura y sus relaciones se pueden describir y explicar según los constructos teóricos de la rección sintáctica y mando inmediato.

Figura 4. Representación sintáctica de predicación primaria y secundaria (SPr)

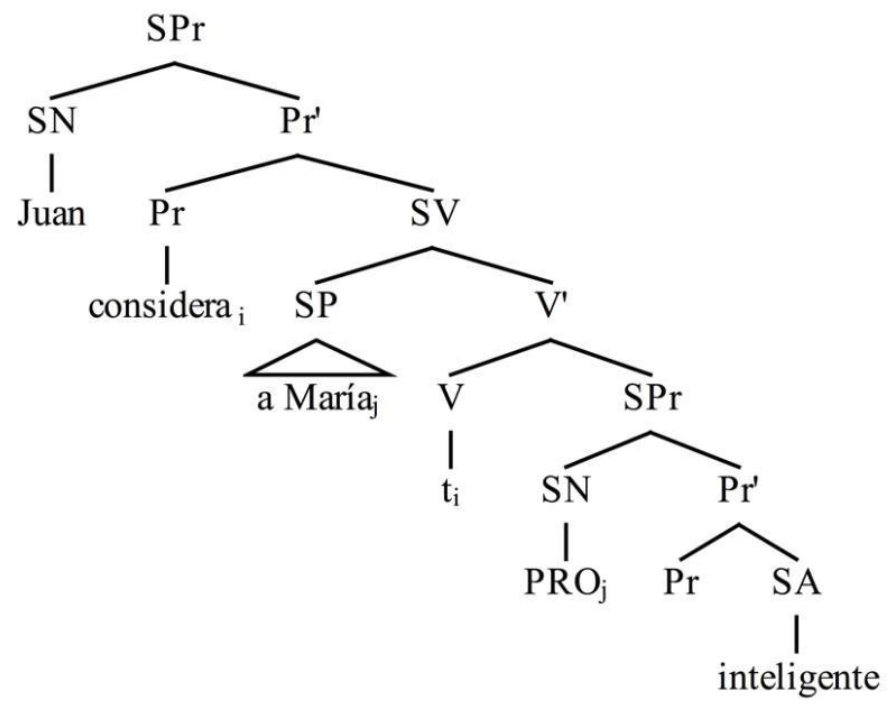




\section{Discusión}

Sin lugar a dudas, la predicación es una función gramatical fundamental en la organización de la gramática de la lengua, pues es el mecanismo que determina cómo se relacionan internamente los constituyentes de la estructura oracional, permitiendo la expresión del significado.

La propuesta generativista tradicional y versiones más recientes han tenido éxito en la descripción y explicación de esta función. Por un lado, se distingue a la predicación primaria o clausular de la secundaria en base a la presencia de un sujeto externo al cual el predicado o verbo principal se refiere a la vez que lo selecciona como parte de su estructura argumental para completar su expresión. En este proceso de selección semántica, se proyectan los rasgos léxicos en la estructura sintáctica en la medida que se satura paralelamente la estructura argumental impuesta por la matriz verbal.

En cuanto a la predicación secundaria, si bien ha sido descrita en español, aún existe una cierta tensión sobre cómo se representa y analiza sintácticamente. Con respecto a lo anterior, la propuesta de Demonte y Masullo $(1987,1999)$ ofrece una descripción y clasificación detallada del fenómeno en español en base a criterios semánticos y en consideración de su comportamiento interlingüístico. Es necesario señalar que gran parte de la evidencia y descripción de los predicados secundarios proviene de la lengua inglesa. Por ejemplo, en inglés se da un tratamiento profundo a estructuras causativas del tipo (1) The gardener watered the tulips flat. Sin embargo, esta estructura no encuentra una correspondencia directa en español y se reduce principalmente a verbos de soporte pseudocausativos como poner y hacer en (2) La sorpresa puso contento/hizo feliz al niño. En (2), el SV puso contento/hizo feliz al niño corresponde a un predicado complejo que selecciona composicionalmente al sujeto externo la sorpresa y le asigna el papel temático de causa. En este caso, cabe preguntarse cómo se forman estos predicados complejos: ¿se derivan de una operación léxica o de una operación exclusivamente estructural?

En cuanto a la representación sintáctica de la predicación secundaria, Demonte y Masullo (1999) sostienen que la estructura corresponde a una cláusula mínima o reducida. Este criterio ha servido para distinguir entre complementos predicativos (seleccionados por el predicado principal) y adjuntos (no seleccionados). No obstante, su posición relativa en el árbol sintáctico no explica cómo se jerarquiza e integra a la proyección máxima en casos de ambigüedad:

María pintó a la modelo descalza.

En el ejemplo, el depictivo descalza podría orientarse al sujeto externo u objeto directo interno. Intuitivamente, se infiere que corresponde a un elemento no seleccionado por el verbo y que, por lo tanto, corresponde a un adjunto. Sin embargo, si se orienta al objeto directo, formaría parte de un predicado complejo. En este punto, surge la interrogante sobre cómo analizar sintácticamente esta construcción gramatical y esquematizarla en un árbol según la propuesta de Demonte y Masullo y que a su vez cumpla con el criterio de rección y condiciones de buena formación. En este caso, corresponde utilizar el SFlex como sigue en la figura 5: 
Figura 5. Representación sintáctica de descalza como CM.

(5a)

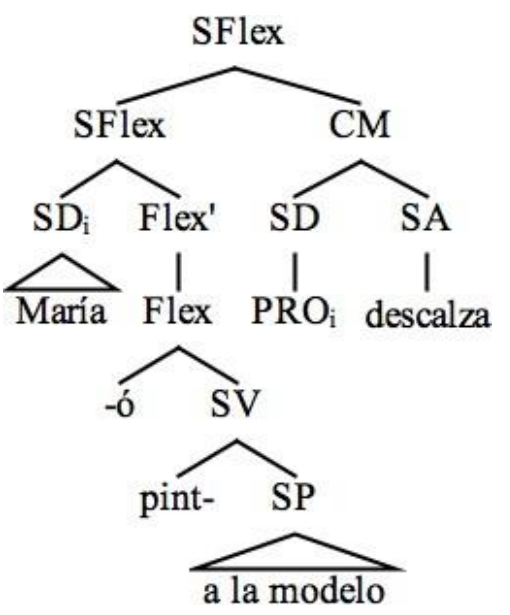

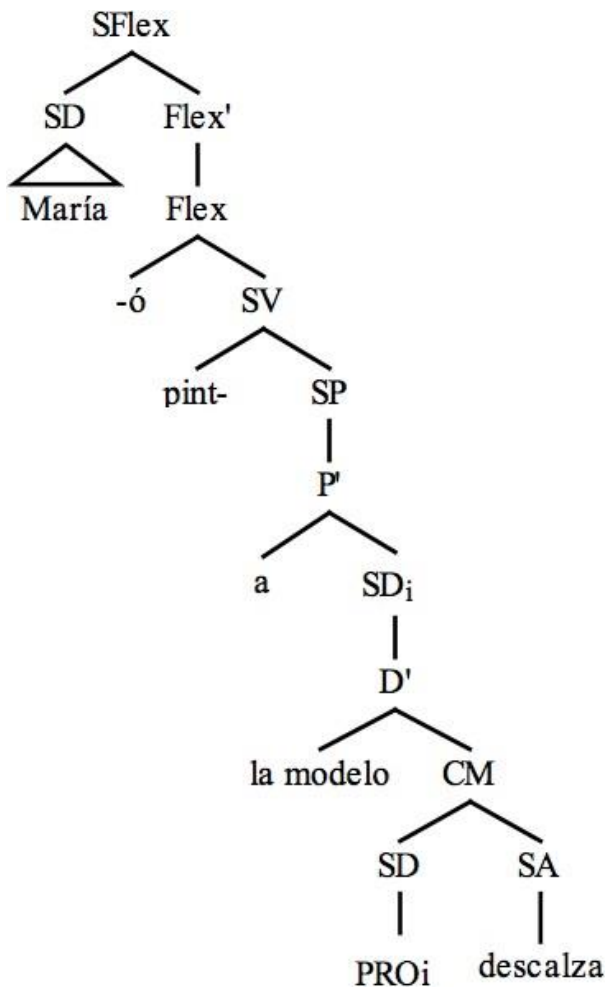

$(5 b)$

(5a) y (5b) representan las posibles interpretaciones de (3). Ambos casos cumplen con el criterio de rección. En (5a) la CM se orienta al sujeto y PROi está controlado por SDi. En (5b) la CM se orienta al objeto directo interno y PROi está controlado por el SDi. Si bien ambos modelos describen relaciones de predicación secundaria, la predicación primaria aparentemente se deriva en paralelo a la CM en (5a). La interpretación de (5b) también es plausible desde el punto de vista estructural. En este caso, el SV y la CM forman una predicación compleja que en conjunto seleccionan al argumento externo. De este modo, la predicación primaria pareciera ser un operador lógico que comanda todo el despliegue de la secuencia y agota su propia función en la medida que integra el contenido proposicional que contiene la CM.

En contraste a la propuesta anterior, Bowers señala que ambas funciones (predicación primaria y secundaria) pueden describirse y explicarse en términos de una función única, a saber, la categoría funcional Pr. Tal como señala Bowers (2001, p. 304):

"Not only does hypothesizing the category Pr unify MC and SC Predication, providing a purely structural characterization of the predication relation, but it also solves the related problem of what category to assign SCs to: a SC is simply the maximal projection of Pr".

En esta perspectiva, Bowers postula que los dos tipos de predicación se pueden representar como una sola función y que esta operación resuelve el problema sobre a qué categoría asignar la CM. Así, el ejemplo (3) se representaría sintácticamente como sigue:

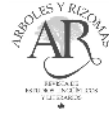


Figura 6. Representación sintáctica de descalza como SPr.

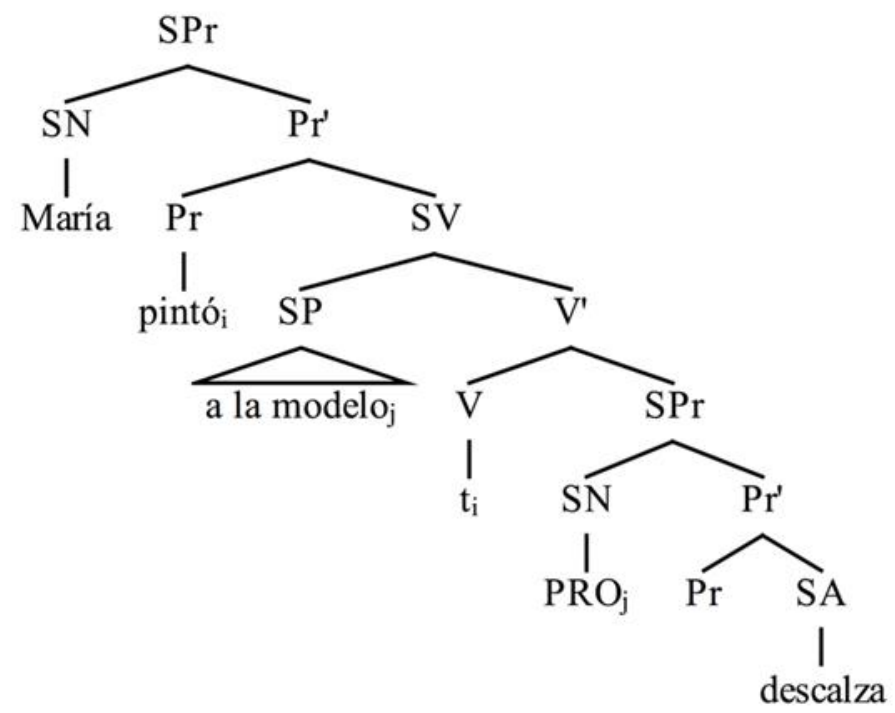

La figura 6 muestra cómo se integra estructuralmente la cláusula mínima, o SPr en términos de Bowers, que corresponde a una relación de predicación secundaria optativa o adjunto. Los argumentos se organizan secuencialmente según los criterios semánticos que impone la estructura interna del predicado principal pintó. Aparentemente, las condiciones semánticas que impone el verbo se proyectan en forma descendiente y en cascada en los slots de la estructura, operación que permitiría explicar por qué el SA descalza se adjunta a la estructura y no se integra en ella. La combinación de descalza y su antecedente controlador inmediato a la modelo retroalimenta semánticamente al predicado principal hasta saturar su expresión a través de una operación ascendente.

En vista del planteamiento anterior, es pertinente señalar que la propuesta de Bowers ofrece una adecuación descriptiva y explicativa de un fenómeno cuya naturaleza desborda la lógica y los postulados desde una perspectiva exclusivamente formal de la gramática generativa tradicional. En el caso de oraciones que presentan ambos tipos de predicación, la categoría funcional Pr permite representar y analizar sintácticamente según criterios semánticos definidos cómo se ejecuta la función predicativa y los tipos de relaciones que se establecen entre los distintos constituyentes de la estructura oracional, especialmente, en casos de relaciones asimétricas como la adjunción.

Para comprobar o refutar la propuesta de Bowers y la lógica que subyace a la representación sintáctica de la predicación primaria y secundaria (obligatoria u optativa), la función predicativa podría modelarse computacionalmente tras someterla a pruebas de adecuación interlingüística y electrofisiológicas, puesto que se trata de un mecanismo lógico humano para la expresión del significado. Esto indudablemente debe complementarse con la capacidad descriptiva de la propuesta de Demonte y Masullo (1999) y otros autores que abordan el fenómeno desde otras perspectivas teóricas, como Rothstein (2011), quien ofrece una mirada desde la semántica formal.

Si bien la representación sintáctica por medio de esquemas de árboles es una abstracción sobre cómo opera la predicación, esta información debiese al menos ofrecer una base teórica desde la lingüística para predecir su representación en el cerebro humano. En este sentido, Sprouse (2013) señala que la teoría sintáctica constituye la teoría computacional del "procesador

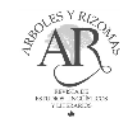


humano de oraciones", pues busca describir las propiedades fundamentales de las oraciones que representan el input para la comprensión y el output para la producción. En otras palabras, la teoría sintáctica debiese aportar información algorítmica que pueda implementarse en un nivel neurolingüístico. De este modo, una violación sintáctica en la representación de la predicación secundaria, por ejemplo, en la concordancia gramatical de persona, número o género debiese activar una respuesta cerebral relacionada a dicho evento. En una oración como María pintó a la modelo desnudo, se esperaría una activación del componente P600 (syntactic positive shift) que ocurre entre 500-800 ms post-estímulo y representa una deflexión positiva frente a violaciones sintácticas de concordancia gramatical y ambigüedad sintáctica (Sprouse, 2013; De Groot \& Hagoort, 2018). En este caso, el morfema gramatical - $O$ no concuerda en género con el referente inmediato ni sujeto externo. Una activación del P600 sugeriría que la integración de la información que expresa la flexión gramatical es determinante en la composición de predicados complejos como pintó a la modelo desnudo y, por extensión, la función predicativa estaría condicionada por la sintaxis. En resumen, el fenómeno linguiístico de la predicación secundaria ofrece un potencial investigativo experimental que sin duda robustecería la teoría sintáctica y nos permitiría profundizar nuestro entendimiento sobre cómo ocurre su procesamiento en tiempo real.

\section{Conclusiones}

En este trabajo, nos propusimos contrastar dos propuestas generativistas que han abordado la predicación secundaria en español. Por un lado, Demonte y Masullo (1999) han descrito formalmente este fenómeno y proporcionado abundante evidencia que permite establecer tipologías que complementan la adecuación explicativa que ofrece la categoría funcional Pr con respecto a su representación y análisis sintáctico-semántico. Así, la propuesta de Bowers $(1993,2001)$ se posiciona como un sistema de análisis pertinente que aúna las relaciones primarias y secundarias en una función predicativa única que está regida por las condiciones que impone el modelo generativista de Chomsky (1981), a saber, criterio de papeles temáticos, rección y X con Barra. Lo anterior permite representar y analizar la función predicativa secundaria, sea esta opcional $\mathrm{u}$ obligatoria, por medio de una estructura jerárquica generativa que transparenta las relaciones lógico-semánticas que se establecen en la estructura argumental y que finalmente se proyectan la estructura superficial de la oración.

Dada la complejidad que presentan las relaciones de predicación secundaria en español en cuanto a su formación por composición, es posible afirmar que su estudio bajo condiciones experimentales controladas podría aportar evidencia empírica sobre cómo se representa en el cerebro humano, específicamente, a través de métodos electrofisiológicos como EEG. Nuestra hipótesis al respecto es que una violación sintáctica en concordancia de género o ambiguiedad en la estructura predicativa secundaria activará una respuesta cerebral del tipo P600, deflexión positiva asociada a este tipo de incongruencias sintácticas.

Finalmente, la predicación secundaria en español debiese recibir mayor atención desde el punto de vista descriptivo y experimental para extender sus alcances a estructuras poco estudiadas como predicados intransitivos pseudocausativos como El niño cayó enfermo. Esto contribuirá a la teoría sintáctica general. 


\section{Referencias}

Bosque, I. \& Gutiérrez, J. (2009). Fundamentos de sintaxis formal. Madrid: Ediciones AKAL. Bowers, J. (1993). The Syntax of Predication. Linguistic Inquiry, 24: 591-656.

Bowers, J. (2001). Predication. En M. Baltin y C. Collins (Eds). Handbook of Contemporary Syntactic Theory (pp. 299-333). Oxford: Wiley-Blackwell.

Bowers, J. (2010). Arguments as Relations. MIT Press, Cambridge, MA.

Carnie, A. (2002). Syntax. A generative introduction. Blackwell Publishing: Oxford.

Chomsky, N. (1965). Aspects of the Theory of Syntax. Cambridge: MIT Press.

Chomsky, N. (1981). Lectures on Government and Binding. Cambridge: MIT Press.

De Groot, A. \& Hagoort, P. (Eds.). (2018). Research methods in psycholinguistics and the neurobiology of language: A practical guide. Oxford: Wiley.

Demonte, V. (1987). C-Command, Prepositions and Predication. Linguistic Inquiry, 18: $147-157$.

Demonte, V. \& Masullo, P. (1999). La predicación: los complementos predicativos. En I. Bosque y V. Demonte (Eds.) Gramática descriptiva de la lengua española. Madrid: Espasa Calpe.

Espinal, M. (coord.), Maciá, J., Mateu, J., \& Quer, J. (2014). Semántica. Madrid: Ediciones AKAL.

Rothstein, S. (2011). Secondary Predicates. En P. Portner, C. Maienborn y K. von Heusinger (Eds.). Semantics: An International Handbook of Natural Language Meaning, (pp. 1442-1462), Vol. 2. Berlin: Mouton.

Schultze-Berndt, E. (2017). Depictive Secondary Predicates in Typological Perspective. En M. Everaert and H. C. Riemsdijk (Eds.). The Wiley Blackwell Companion to Syntax. Hoboken, NJ: John Wiley \& Sons, Inc.

https://doi.org/10.1002/9781118358733.wbsyncom115

Sprouse, J. \& Lau, E. (2013). Syntax and the brain. En Den Dikken, M. (Ed.) The Cambridge Handbook of Generative Syntax, Cap. 26. Cambridge: Cambridge University Press. https://doi.org/10.1017/cbo9780511804571.033 